\title{
Stable dual-wavelength fiber laser utilizing tapered-EDF as comb filter in hybrid Raman-EDF gains
}

\begin{abstract}
A dual-wavelength hybrid Raman-erbium doped fiber ring laser is proposed and demonstrated. By tapering a section of erbium doped fiber ends with two-taper successively, the fiber laser can lase two wavelengths at $1563.08 \mathrm{~nm}$ (laser 1) and $1589.5 \mathrm{~nm}$ (laser 2). This dual wavelengths output exhibits a good performance having the side mode suppression ratio over $52 \mathrm{~dB}$ and acceptably high peak power at $-3.2 \mathrm{dBm}$ for both. The lasers also shows high stability at room temperature with peak power variations of less than $0.29 \mathrm{dBm}$ for laser 1 and $0.39 \mathrm{dBm}$ for laser 2 and wavelength drift less than $0.04 \mathrm{~nm}$ when observed up to 2 hours of operations.
\end{abstract}

Keyword: Tapered-EDF; Comb filter; Mach-Zehnder interferometer; Dual-wavelength laser 